International Journal of Risk \& Safety in Medicine 1 (2020) 1-10

\title{
Determinants of sleep habits in toddlers: Application of planned behavior theory
}

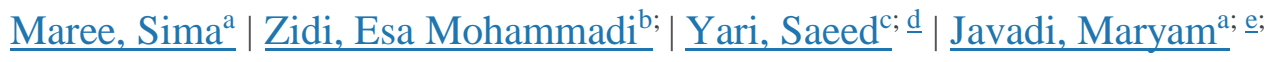

Affiliations: [a] Children Growth Research Center, Research Institute for Prevention of NonCommunicable Diseases, Qazvin University of Medical Sciences, Qazvin, Iran | [b] Department of Public Health, School of Health, Qazvin University of Medical Sciences, Qazvin, Iran | [c] Student Research Committee, Department of Health, Shahid Beheshti University of Medical Sciences, Tehran, Iran | [d] Department of Occupational Health Engineering, Faculty of Health, Neyshabur University of Medical Sciences, Neyshabur, Iran | [e] Department of Nutrition, School of Health, Qazvin University of Medical Sciences, Qazvin, Iran.

Correspondence: [*] Address for correspondence: Maryam Javadi, Associate Professor (Ph.D. in Nutrition), Children Growth Research Center, Research Institute for Prevention of NonCommunicable Diseases, Qazvin University of Medical Sciences, Qazvin, 34197-59811, Iran. Tel.: +989121145572; E-mail: mjavadi@qums.ac.ir, mz_javadi46@yahoo.com. Esa Mohammadi Zidi, Associate Professor (Ph.D. in Nutrition), Department of Nutrition, School of Health, Qazvin University of Medical Sciences, Qazvin, 34159-14595, Iran. Tel.: +989124146500; Email: easamohammadizeidi@gmail.com

\begin{abstract}
Background: Sleep health is a prerequisite for mental and physical health. The family affects the sleeping habits of the child.

Objective: The purpose of this study was to investigate the theory of mothers' planned behavior in predicting sleeping habits of toddlers.

Methods: The participants of this descriptive-analytical study were 120 mothers of 12 - to 36month-old children living in Razan, Hamadan province, Iran. Data were collected in three stages in order to develop a theory-based tool. Semi-structured qualitative interviews with mothers were conducted to investigate the application of theory in predicting mothers' intention and behavior $(n$ $=25$ ). The face and content validity of the questionnaire was confirmed by a panel of experts. Testretest method was used to determine the reliability of the questionnaire at 3 weeks interval $(n=$ 25). Validity of Structural Convergence and Divergence with Factor Analysis, Internal Consent with Cronbach's Alpha and predictive validity was determined by multiple regression coefficients $(n=120)$.

Results: The mean age of the children was $22.8 \pm 7.5$ months, $55 \%$ (66 children) were boys, the mean score of the children's sleep questionnaire was $49 \pm 6$, and the children's sleep duration was $9 \pm 1$ hour. Theoretical constructs have a positive and direct correlation with each other and with children's sleep habits, which demonstrates the validity of the theory in predicting toddler sleep habits. Regression analysis showed that perceived behavioral control was the strongest predictor
\end{abstract}


of sleeping habits of toddlers $(\beta=0.446)(P=0.000)$. The theory of planned behavior $(\mathrm{TPB})$ is able to explain 30.5 to $25.7 \%$ of the variance of toddler sleep habits.

Conclusion: While recommending more comprehensive studies, the findings of the study emphasize the application of the theory of planned behavior in identifying factors influencing sleep habits and designing child sleep improvement interventions 